\title{
Spinal anesthesia-induced hypotension is caused by a decrease in stroke volume in elderly patients
}

This article was published in the following Dove Medical Press journal: Local and Regional Anesthesia

\author{
Charlotte Hofhuizen' \\ Joris Lemson' \\ Marc Snoeck ${ }^{2}$ \\ Gert-Jan Scheffer ${ }^{3}$ \\ 'Department of Critical Care, \\ Radboud University Medical \\ Center, Nijmegen, The Netherlands; \\ ${ }^{2}$ Department of Anesthesia, Canisius- \\ Wilhelmina Ziekenhuis, Nijmegen, \\ The Netherlands; ${ }^{3}$ Department of \\ Anesthesia, Radboud University \\ Medical Center, Nijmegen, The \\ Netherlands
}

Background: Hypotension is common during spinal anesthesia (SA) and is caused by a decrease in systemic vascular resistance (SVR) and/or cardiac output (CO). The effect of the dose of bupivacaine administered intrathecally on the changes in $\mathrm{CO}$ in elderly patients is largely unknown. This study investigated the hemodynamic effect of SA in elderly patients by studying the effect of two different dosages of intrathecal bupivacaine.

Methods: This prospective cohort study included 64 patients aged $>65$ years scheduled for procedures under SA; the patients received either $15 \mathrm{mg}$ bupivacaine (the medium dose [MD] group) or $10 \mathrm{mg}$ bupivacaine and $5 \mu \mathrm{g}$ sufentanil (the low dose [LD] group). Blood pressure and $\mathrm{CO}$ were monitored throughout the procedure using Nexfin ${ }^{\mathrm{TM}}$, a noninvasive continuous monitoring device using a finger cuff.

Results: Thirty-three patients received MD and 31 received LD and there was no mean difference in baseline hemodynamics between the groups. On an average, the CO decreased $11.6 \%$ in the MD group and $10.0 \%$ in the LD group. There was no significant change in SVR. Incidence of a clinically relevant decrease in stroke volume (SV) (>15\% from baseline) was $67 \%$ in the MD and $45 \%$ in the LD groups $(P<0.05)$.

Conclusion: $\mathrm{CO}$ and blood pressure decreased significantly after the onset of SA in elderly patients. This is mainly caused by a decrease in SV and not by a decrease in SVR. There was no difference in $\mathrm{CO}$ and blood pressure change between dosages of 10 or $15 \mathrm{mg}$ bupivacaine.

Keywords: cardiac output, intrathecal anesthesia, local anesthetics, dose, finger blood pressure measurement

\section{Introduction}

Hypotension is a common side effect of spinal anesthesia (SA) and it occurs in 16-33\% of cases. ${ }^{1}$ This response is exaggerated in the elderly where a negative influence on a relatively higher resting sympathetic tone and decreased baroreceptor activity may explain the higher incidence of hypotension in response to SA. ${ }^{2,3}$ Hypotension after the onset of SA is thought to be caused by either a decrease in systemic vascular resistance (SVR) or cardiac output (CO) or both.

Until recently, $\mathrm{CO}$ measurement required invasive monitoring, which is unsuitable in awake patients having SA for short procedures. Therefore, studies regarding factors that might influence the hemodynamic effect of SA on $\mathrm{CO}$, eg, doses of intrathecal local anesthetics, are limited. ${ }^{4,5}$ Moreover, most CO monitors produce only intermittent measurements thereby providing only limited understanding of fast hemodynamic changes.
Correspondence: Charlotte Hofhuizen Department of Critical Care, Radboud University Medical Center, PO Box 910I, Nijmegen 6500 HB, Nijmegen, The Netherlands

Tel +3I $2436 \mid 7273$

Fax +3I $243635 I 56$

Email charlotte.hofhuizen@radboudumc.nl 
Nexfin $^{\mathrm{TM}}$ (Edwards Lifesciences, Irvine, CA, USA) provides a continuous, noninvasive blood pressure and CO measurement using a finger cuff. This device does not bring inconvenience to patients making it clinically suitable for monitoring awake patients having regional anesthesia. Several recent studies have shown the device to be sufficiently accurate in the measurement of absolute $\mathrm{CO}$ and blood pressure values as well as in tracking $\mathrm{CO}$ and blood pressure changes. ${ }^{6-8}$

In this study we have investigated the hemodynamic effects of SA during uncomplicated surgical procedures. Since we hypothesize that the hemodynamic effects would be dose-dependent, we evaluated two different dosages of local anesthetics to provide a better understanding of the underlying cause of hypotension after the onset of SA.

\section{Patients and methods}

\section{Patients}

We performed a prospective double cohort study. The Regional Ethics Committee in Nijmegen, the Netherlands, responsible for medical research in humans (Ethical Committee No. 20091171) approved the study and the need for informed consent was waived because of the purely observational nature of the study. The research was performed in accordance with the Declaration of Helsinki. Participants recruited were individuals admitted for elective surgery to a 500-bed teaching hospital in Nijmegen, the Netherlands. The study period was from December 2009 to January 2011. Seventy-one consecutive patients, American Society of Anaesthesiologists class I-III, aged $\geq 65$ years scheduled for elective orthopedic, general, or vascular surgery using SA were included. Patients with cardiac arrhythmia, digital ischemia, allergic reactions to opiates or local anesthetics, perioperative blood loss that exceeded $1 \mathrm{~L}$, and New York Heart Association class III-IV congestive heart failure were excluded from the study.

\section{SA}

Since the main focus of this study was to evaluate the hemodynamic effect of SA, we performed an observational study in patients receiving local anesthetics intrathecally. To provide a better understanding of their effect on hemodynamics, we observed two different intrathecal dosage groups. For the medium dose (MD), we included patients receiving $15 \mathrm{mg}$ bupivacaine intrathecally. For the low dose (LD), we chose to study the lowest intrathecal dose of bupivacaine that would with great probability still provide effective anesthesia for certain long procedures such as hip surgery or vascular surgery. Five microgram Sufenta ${ }^{\mathrm{TM}}$ was regularly added to the
LD to ensure adequate intensity and duration of analgesia and to administer an identical volume intrathecally. ${ }^{9}$ We compared patients receiving $10 \mathrm{mg}$ bupivacaine with $5 \mu \mathrm{g}$ Sufenta ${ }^{\mathrm{TM}}$ to patients receiving 'medium' intrathecal dose of $15 \mathrm{mg}$ bupivacaine. Extension of anesthesia to the higher thoracic dermatomes may lead to bradycardia due to blockage of the cardiac sympathetic accelerator fibers arising from the first four thoracic segments. To avoid this, as a high spinal block would surely have an effect on $\mathrm{CO}$, SA was performed at L2-3 or L3-4 and never at a higher interspace. ${ }^{10}$ For clarity, we named the $10 \mathrm{mg}$ bupivacaine regime as LD and the 15 mg bupivacaine regime as MD. The dose of bupivacaine, $\mathrm{LD}$ or MD, was based upon the clinical decision of the attending anesthesiologist based on patient characteristics and personal preference, and there was no randomization. The research team was blinded for the dose administered intrathecally.

\section{Study protocol}

One hour before surgery, patients received $1,000 \mathrm{mg}$ acetaminophen and anxious patients received either $7.5 \mathrm{mg}$ midazolam or $10 \mathrm{mg}$ oxazepam orally. The patients were fasted overnight and oral fluid intake was allowed for up to 2 hours before the procedure. Neither IV fluid was infused before entering the study, nor were any prophylactic vasoactive drugs administered (like, ephedrine or atropine). After arrival in the operating room intravenous access was established. Perioperative hemodynamic monitoring included the noninvasive measurement of heart rate (HR) using electrocardiographic recording electrodes, noninvasive blood pressure using oscillometry, and a pulse oximeter. The noninvasive arterial blood pressure $\left(\mathrm{ABP}_{\mathrm{N}}\right)$ and $\mathrm{CO}$ measurement $\left(\mathrm{CO}_{\mathrm{NI}}\right)$ were obtained using a finger cuff adjusted to the size of the index finger of the patient according to the guidelines of the manufacturer and connected to the Nexfin ${ }^{\mathrm{TM}}$ monitor. This noninvasive device is based on the principle of Peňaz and measures the diameter of the finger's artery using an inflatable finger cuff and a built-in photoelectric plethysmograph. ${ }^{11}$ From this signal, blood pressure in the brachial artery is reconstructed. Nexfin ${ }^{\mathrm{TM}}$ calculates beat-to-beat $\mathrm{CO}$ by dividing the area under the systolic portion of the arterial pressure curve by the aortic input impedance, which is determined from a three-element Windkessel model described by Westerhof et $\mathrm{al}^{12}$ using the influence of the patient's age, height, weight, and sex on aortic mechanical properties.

SA was performed with the patient in sitting position, a 27-gauge pencil-point needle was inserted in the subarachnoid space at the L2-3 or L3-4 interspace. After obtaining free flow of clear cerebrospinal fluid either $3 \mathrm{~mL}$ bupivacaine, 
$5 \mathrm{mg} / \mathrm{mL}(\mathrm{MD})$ or an admixture of $2 \mathrm{~mL}$ bupivacaine $0.5 \%$ and $1 \mathrm{~mL}$ sufentanil, $5 \mu \mathrm{g} / \mathrm{mL}$ (LD) was injected with the spinal needle bevel facing cephalad. Immediately after injection, patients were placed in the supine position.

Hypotension was defined as a decrease in mean arterial pressure (MAP) $>25 \%$ from baseline value or systolic arterial pressure $(\mathrm{SAP}<100 \mathrm{mmHg}$, and was treated with $5 \mathrm{mg}$ IV bolus ephedrine and repeated every 3 minutes until the hypotension resolved. Bradycardia was defined as a $\mathrm{HR}<40$ beats per minute and was treated with atropine $0.5 \mathrm{mg}$ IV. During surgery, isotonic saline $0.9 \%$ solution was infused at a rate of $1.5 \mathrm{~mL} / \mathrm{kg} /$ hour. If blood loss exceeded $300 \mathrm{~mL}$ this was compensated using hydroxyethyl starch solution (130/0.46\% hydroxyethyl starch solution; Fresenius Kabi, Bad Homburg vor der Höhe, Germany).

\section{Measurements}

A baseline $\mathrm{ABP}_{\mathrm{NI}}$ and $\mathrm{CO}_{\mathrm{NI}}$ measurements were performed during 3 minutes in supine position before commencing the SA procedure. Hemodynamic data were recorded and stored electronically using the Nexfin ${ }^{\mathrm{TM}}$ and analyzed offline. After performance of SA, the patient was repositioned in supine position and $\mathrm{ABP}_{\mathrm{NI}}$ and $\mathrm{CO}_{\mathrm{NI}}$ were measured continuously until the end of surgery as measurements beyond this point, during transport to the recovery room, etc, would introduce movement artifacts and bias due to replacement of the finger cuff. The sensory block level was tested at 5-minute intervals using a cold discrimination. Time to discharge from the recovery room to the ward, according to the Post Anesthesia Care Unit protocol after regression of sensory level to Th12, Aldrete score $\geq 9$ and NRS $<4$, was recorded. ${ }^{13}$

\section{Statistical analysis}

A decrease in stroke volume (SV) is one of the most important possible sources of hypotension after SA. However, to our knowledge, there are no studies regarding the effect of the dosage of intrathecal local anesthetics on SV, and therefore, we used a composite outcome parameter, $\mathrm{CO}$, as the primary effect parameter 30 minutes after the onset of anesthesia. Based on a previous study we expected the difference in $\mathrm{CO}$ between the two study groups to be $0.75 \mathrm{~L} / \mathrm{min} .{ }^{5}$ We calculated that a total sample size of 60 patients would allow us to detect this CO difference based on a SD of $20 \%$ and a $5 \%$ type 1 error risk. ${ }^{14}$ To allow for potential dropouts from treatment, we included 71 patients. A decrease of $>25 \%$ in MAP or SAP or a decrease in $\mathrm{SV}>15 \%$ was considered clinically significant.

A MedCalc ${ }^{\circledR}$ software package (MedCalc Software, Ostend, Belgium) was used for statistical analysis. Patient characteristics are expressed as mean and range, and hemodynamic data as mean and SD. Assumption of normality was checked using a Kolmogorov-Smirnov test. To compare demographic and hemodynamic data from baseline Student's t-test, chi-squared test, and one-way ANOVA were used. $P<0.05$ was considered statistically significant.

Hemodynamic variables were stored on the Nexfin ${ }^{\mathrm{TM}}$ monitor and analyzed offline. We used 30-second time intervals to analyze hemodynamic effects of SA that were sampled at various intervals during the surgical procedure until 40 minutes after the onset of SA.

\section{Results}

Seventy-one patients were included in the study. In three patients a good quality noninvasive finger signal could not be obtained. Four patients were excluded from further analysis because perioperative blood loss exceeded $1 \mathrm{~L}$, this resulted in 64 patients with a complete data set (Figure 1). Patient characteristics are depicted in Table 1. The majority of patients underwent hip surgery, either elective replacement or repair after a hip fracture. Data collection ended at the end of surgery with a mean duration of 40 minutes. Two patients required supplemental intravenous analgesia, one in each study group. Baseline patient characteristics and hemodynamic values did not differ statistically between the two groups (Table 2).

The course of changes in SV after initiation of SA is depicted in Figure 2. Mean hemodynamic changes that occurred during the course of measurements are depicted in Table 3. SV decreased $11 \%(P<0.001)$ in the MD group and decreased $8 \%$ in the LD group $(P=0.01)$. An SV decrease of $>15 \%$ occurred in $67 \%$ and $45 \%$ of patients in the MD and the LD group, respectively (Table 4).

Mean dosage of ephedrine was $7.8 \mathrm{mg}$ in the MD group and $4.7 \mathrm{mg}$ in the LD group $(P=0.14)$. Three patients in the MD group required rescue medication (phenylephrine) to maintain blood pressure, no patients in the LD group had hypotension unresponsive to ephedrine. Baseline hemodynamic values did not differ between patients who did and did not require ephedrine (Table 5). Changes in blood pressure, $\mathrm{HR}$, and $\mathrm{CO}$ were not influenced by blood loss or the height of sensory block.

\section{Discussion}

Blood pressure decreases significantly after onset of SA. In this study, this was caused by a decrease in $\mathrm{CO}$ and not by a decrease in SVR. There was no difference in mean decrease in $\mathrm{CO}$ and MAP between the two dosage groups. Nexfin ${ }^{\mathrm{TM}}$ 


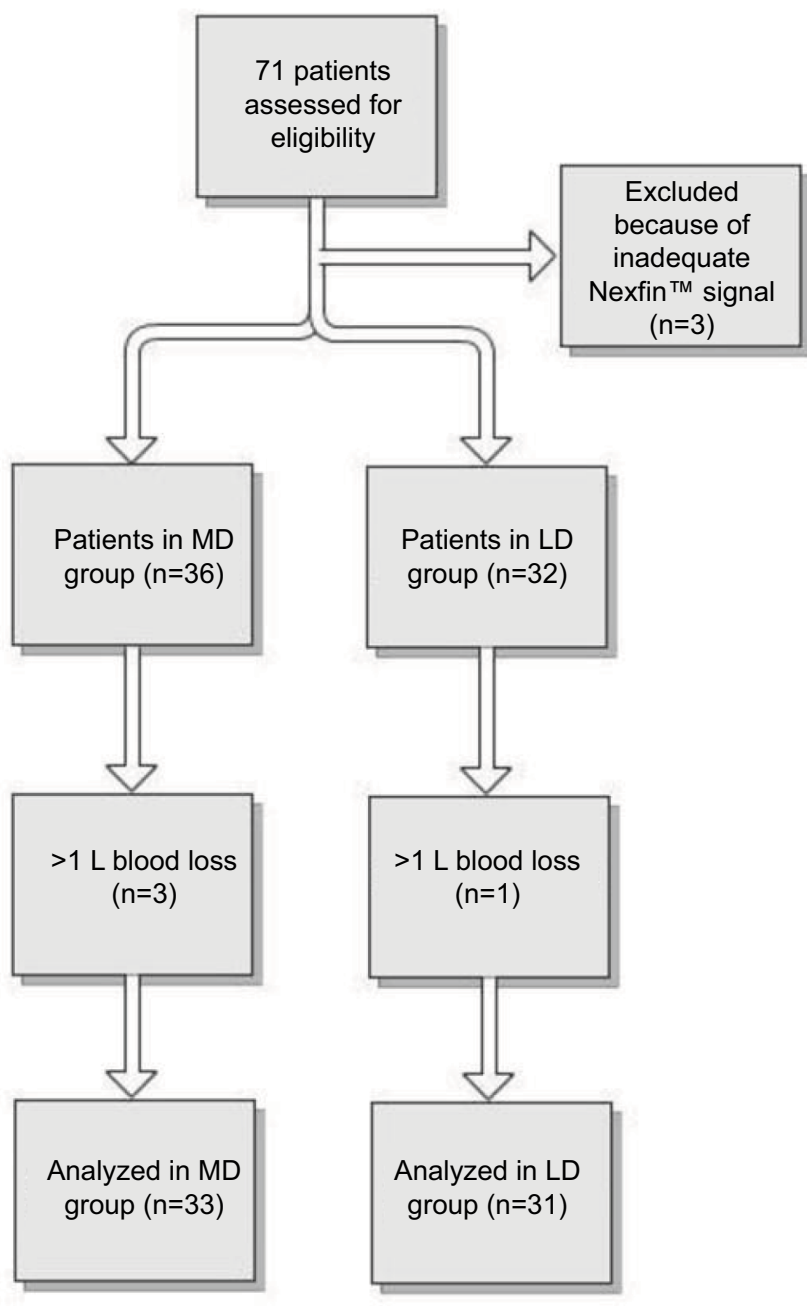

Figure I Flow diagram of trial procedure.

Note: The patients with >I L blood loss were excluded.

Abbreviations: LD, low dose; MD, medium dose. proved to be suitable to demonstrate different hemodynamic effects of two spinal anesthetic regimes.

Several mechanisms are proposed to be the cause of the hypotensive response after SA. First, sympathetic blockage from $\mathrm{T} 1$ to $\mathrm{L} 2$ with subsequent arteriolar vasodilation leads to a reduction in SVR, contributing to intraoperative hypotension. This decrease in SVR is often thought to be the main cause of hypotension after SA. Second, a decrease in venous vasomotor tone increases venous pooling and consequently reduces venous return, thereby decreasing CO. Finally, the physiological hemodynamic reserve capacity decreases with age, and limited cardiovascular compensation mechanisms contribute to a decline in $\mathrm{CO}$ and blood pressure in response to SA. ${ }^{15}$

After the onset of SA, CO decreased in both groups as a result of a decrease in SV. The incidence of a clinically relevant decrease in SV and SAP was higher in the MD group compared to the LD group. Since blood pressure (afterload) decreased and a change in cardiac muscle contractility was unlikely, the only explanation for the decrease in SV was a reduction in venous return. ${ }^{16,17}$ Indeed, a study in dogs showed that SA causes a decline in mean systemic filling pressure (MSFP). This decrease in MSFP is caused by a redistribution of blood volume to the splanchnic vasculature and to the lower extremities as a result of sympathetic 'denervation' induced by local anesthetics used for SA. ${ }^{3,18}$ This causes a reduction in venous return, and therefore, a decrease in $\mathrm{SV}^{19,20}$

This decrease in SV was not compensated by an increase in HR to maintain CO. Possible explanations are a blunted $\beta$-adrenoreceptor response that is observed in elderly

Table I Patient characteristics

\begin{tabular}{|c|c|c|c|}
\hline & MD $(n=33)$ & LD $(n=3 I)$ & $P$-value \\
\hline Age (years), mean (range) & $74(65-89)$ & $74(65-86)$ & NS \\
\hline $\operatorname{Sex}(M / F), n / n$ & $10 / 23$ & $12 / 19$ & NS \\
\hline BMI $\left(\mathrm{kg} / \mathrm{m}^{2}\right)$, mean (range) & $26.6(19.4-35.6)$ & $26.1(20.0-33.5)$ & NS \\
\hline ASA (I/II/III), n/n/n & $4 / 24 / 8$ & $4 / 22 / 5$ & NS \\
\hline \multicolumn{4}{|l|}{ Type of surgery, $n$} \\
\hline Orthopedic & 27 & 26 & \\
\hline Vascular & 3 & I & \\
\hline General & 3 & 4 & \\
\hline Sensory level, mean (range) & Th9 (Th4-ThI2) & Th9 (Th6-LI) & NS \\
\hline Blood loss (mL), mean (range) & $222(30-600)$ & $218(20-500)$ & NS \\
\hline Time in recovery room (min), mean (range) & $94(30-180)$ & $81(30-180)$ & NS \\
\hline Ephedrine (mg), mean (range) & $7.8(0-40)$ & $4.7(0-15)$ & NS \\
\hline Ephedrine, $\mathrm{n}(\%)$ & $19(57.6 \%)$ & $13(41.9 \%)$ & NS \\
\hline Rescue medication, $n$ & 3 & 0 & NS \\
\hline
\end{tabular}

Note: Data are expressed as mean and range unless otherwise stated.

Abbreviations: ASA, American Society of Anaesthesiologists; BMI, body mass index; LD, low dose; MD, medium dose; NS, not significant. 
Table 2 Baseline hemodynamic variables

\begin{tabular}{|l|l|l|l|}
\hline Baseline & MD (SD) & LD (SD) & $P$-value \\
\hline CO (L/min) & $4.32(1.3)$ & $4.49(0.89)$ & NS \\
SV (mL) & $64(16)$ & $67(17)$ & NS \\
MAP (mmHg) & $99(17)$ & $99(16)$ & NS \\
SAP $(\mathrm{mmHg})$ & $141(30)$ & $140(25)$ & NS \\
DAP $(\mathrm{mmHg})$ & $74.5(11.5)$ & $74.6(11.8)$ & NS \\
HR $(\mathrm{b} / \mathrm{min})$ & $68(14)$ & $69(11)$ & NS \\
SVR $($ dyn s/cm $)$ & $1,905(625)$ & $1,838(503)$ & NS \\
\hline
\end{tabular}

Notes: Data are expressed as mean (SD).

Abbreviations: $\mathrm{CO}$, cardiac output; DAP, diastolic arterial pressure; HR, heart rate; LD, low dose; MAP, mean arterial pressure; MD, medium dose; NS, not significant; SAP, systolic arterial pressure; SV, stroke volume; SVR, systemic vascular resistance.

patients, ${ }^{21}$ use of beta-adrenergic blocking medication, or blockade of the sympathetic cardio-accelerator fibers caused by $\mathrm{SA},{ }^{3}$ although very few patients had a peak sensory block height $\geq$ Th5. However, a sympathetic block can extend above 2-6 dermal segments with sensory loss. ${ }^{22}$

Several previous studies have identified a decrease in SVR as the main determinant of hypotension. In these studies patients received fluid loading just before or after the onset of SA. ${ }^{16,18,23}$ This fluid loading can significantly increase stressed volume and, therefore, venous return. In individuals to whom preload is administered as a 'standard procedure', SV and CO remain unchanged after the onset of SA. In our study, patients did not receive any fluid loading, possibly explaining why hypotension was mainly caused by a decrease in SV and not by a decrease in SVR. Also, fluid loading in itself

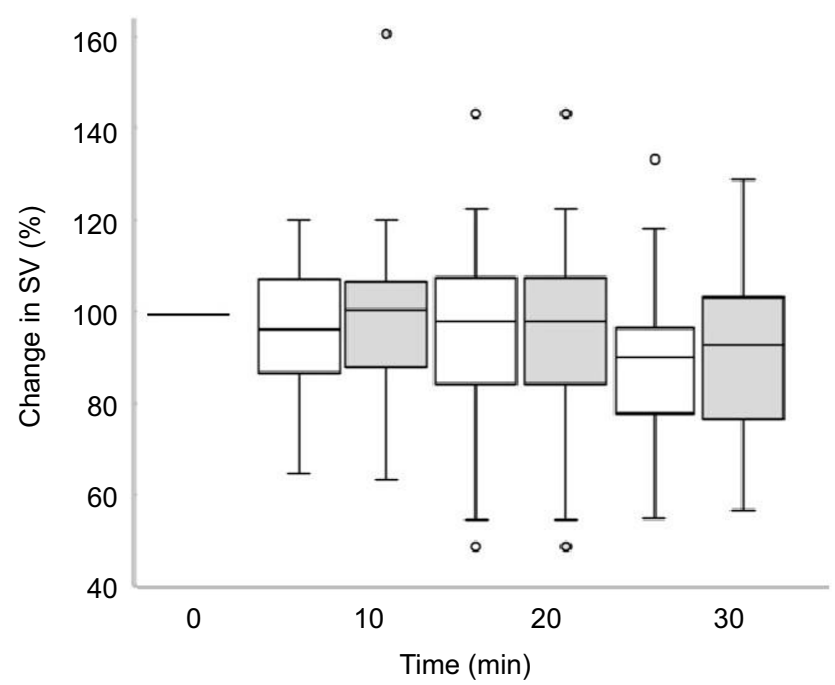

Figure 2 Change of SV in the MD and LD groups at 10, 20, and 30 minutes after SA Notes: Baseline values are represented as $100 \%$, white boxes represent MD, gray boxes represent $L D$, and the dots represent outliers.

Abbreviations: LD, low dose; MD, medium dose; SA, spinal anesthesia; SV, stroke volume.
Table 3 Maximum increases and decreases in hemodynamic variables and mean decrease at 30 minutes after onset of SA

\begin{tabular}{|c|l|l|l|}
\hline & MD (SD) & LD (SD) & P-value \\
\hline CO & & & \\
Percent change at 10 minutes & $5.9(12.1)^{*}$ & $5.3(16.1)$ & NS \\
Percent change at 20 minutes & $10.1(10.9)^{*}$ & $12.4(16.6)^{*}$ & NS \\
Percent change at 30 minutes & $11.6(13.6)^{*}$ & $12.9(15.2)^{*}$ & NS \\
\hline SV & & & \\
Percent change at 10 minutes & $3.3(13.3)$ & $1.5(18.9)$ & NS \\
Percent change at 20 minutes & $7.3(13.8)^{*}$ & $4.9(20.4)^{*}$ & NS \\
Percent change at 30 minutes & $10.7(16.2)^{*}$ & $8.0(18.9)^{*}$ & NS \\
\hline MAP & & & \\
Percent change at 10 minutes & $4.6(14.3)$ & $2.3(11.8)$ & NS \\
Percent change at 20 minutes & $6.5(15.4)$ & $10.4(15.3)^{*}$ & NS \\
Percent change at 30 minutes & $8.4(21.2)^{*}$ & $9.9(15.1)^{*}$ & NS \\
\hline SAP & & & \\
Percent change at 10 minutes & $3.9(15.1)$ & $0.2(15.6)$ & NS \\
Percent change at 20 minutes & $7.6(15.2)^{*}$ & $7.8(17.2)^{*}$ & NS \\
Percent change at 30 minutes & $12.3(20.4)^{*}$ & $9.9(17.8)^{*}$ & NS \\
\hline DAP & & & \\
Percent change at 10 minutes & $0.9(14.9)$ & $0.8(11.8)$ & NS \\
Percent change at 20 minutes & $1.3(16.8)$ & $5.0(13.6)$ & NS \\
Percent change at 30 minutes & $4.9(18.4)^{*}$ & $5.8(13.7)^{*}$ & NS \\
\hline HR & & & \\
Percent change at 10 minutes & $2.2(9.1)$ & $3.9(8.8)$ & NS \\
Percent change at 20 minutes & $1.5(12.2)$ & $6.8(11.8)^{*}$ & NS \\
Percent change at 30 minutes & $1.0(16.0)$ & $3.7(14.9)$ & NS \\
\hline SVR & & & \\
Percent change at 10 minutes & $7.4(27.3)$ & $8.2(23.15)$ & NS \\
Percent change at 20 minutes & $7.5(20.7)$ & $8.6(32.4)$ & NS \\
Percent change at 30 minutes & $5.0(23.0)$ & $5.6(22.9)$ & NS \\
\hline
\end{tabular}

Notes: Data are expressed as mean (SD). $* P \leq 0.05$ compared to baseline. Abbreviations: $\mathrm{CO}$, cardiac output; DAP, diastolic arterial pressure; HR, heart rate; LD, low dose; MAP, mean arterial pressure; MD, medium dose; NS, not significant; SAP, systolic arterial pressure; SA, spinal anesthesia; SV, stroke volume; SVR, systemic vascular resistance.

may cause a decrease in SVR because of hemodilution with a decline in viscosity, which explains why an increase in $\mathrm{CO}$ may not necessarily lead to an increase in blood pressure. ${ }^{24,25}$

The mean decrease in $\mathrm{CO}$ was $11 \%, 40$ minutes after the onset of SA. Other studies reported similar results with $\mathrm{CO}$ decreases ranging from $8 \%-14 \%$ in similar research populations. ${ }^{18,26,27}$ We did not observe a significant differ-

Table 4 Percentage of patients developing clinically significant hemodynamic changes in both study groups

\begin{tabular}{|l|l|l|l|}
\hline & MD & LD & $P$-value \\
\hline SV decrease $>15 \%$ & $23 / 33(67 \%)$ & $14 / 31(45 \%)$ & 0.047 \\
MAP decrease $>25 \%$ & $12 / 33(37 \%)$ & $6 / 31(19 \%)$ & NS \\
SAP decrease $>25 \%$ & $16 / 33(48 \%)$ & $6 / 31(19 \%)$ & 0.014 \\
\hline
\end{tabular}

Abbreviations: LD, low dose; MAP, mean arterial pressure; MD, medium dose; NS, not significant; SAP, systolic arterial pressure; SV, stroke volume. 
Table 5 Baseline hemodynamic values of patients who required ephedrine and patients who did not require ephedrine

\begin{tabular}{|l|l|l|l|}
\hline & With ephedrine (SD) & Without ephedrine (SD) & P-value \\
\hline CO $(\mathrm{L} / \mathrm{min})$ & $4.23(1.0)$ & $4.59(1.15)$ & NS \\
SV $(\mathrm{mL})$ & $63.1(14.7)$ & $68.4(17.6)$ & NS \\
MAP $(\mathrm{mmHg})$ & $99.3(18.1)$ & $100.1(16.2)$ & NS \\
SAP $(\mathrm{mmHg})$ & $140.4(29.2)$ & $142.8(28.4)$ & NS \\
DAP $(\mathrm{mmHg})$ & $75.2(12.7)$ & $74.5(10.7)$ & NS \\
HR $(\mathrm{b} / \mathrm{min})$ & $68.0(13.2)$ & $68.4(11.9)$ & NS \\
SVR $\left(\mathrm{dyn} \cdot \mathrm{s}_{\mathrm{cm}}^{\mathrm{s}}\right)$ & $1,964.9(624.7)$ & $1,819.3(512)$ & NS \\
\hline
\end{tabular}

Notes: Data are expressed as mean (SD).

Abbreviations: CO, cardiac output; DAP, diastolic arterial pressure; HR, heart rate; MAP, mean arterial pressure; NS, not significant; SV, stroke volume; SAP, systolic arterial pressure; SVR, systemic vascular resistance.

ence in changes in $\mathrm{CO}$ between the two dosage groups. This was also found in a previous study in an obstetric population. ${ }^{4}$ A correlation between change in $\mathrm{CO}$ and dosage of subarachnoid bupivacaine-sufentanil has been suggested. ${ }^{5}$ However, in the latter study lower dosages of subarachnoid bupivacaine ( 7.5 and $12.5 \mathrm{mg}$ ) were used, study population was small and a different noninvasive $\mathrm{CO}$ monitor was used, impedance cardiography. The administration of vasopressor medication might blur statistical difference in $\mathrm{CO}$ between the two groups. Vasopressor medication exerts an important part of its action by increasing venous return by increasing MSFP and therefore $\mathrm{CO}^{28,29}$

In this study, hypotension was caused by a decrease in SV. Low SV can be treated with volume therapy; the administration of vasopressor medication likewise increases SV due to an increase in preload. However, a clinical implication of this study can be that if a patient remains hypotensive despite vasopressor medication, administration of intravenous fluid is indicated to increase $\mathrm{SV}$ and consequently blood pressure. This hypothesis is supported by an earlier study in elderly patients undergoing hip surgery SA. Lithium dilution cardiac output (LiDCO, Lido Group, London, UK)-based fluid therapy was applied to optimize SV, patients required very little vasopressor medication as a consequence. ${ }^{30}$ This is further supported by previous studies in obstetric patients, where co-loading, infusing fluid immediately after the onset of SA with either crystalloid or colloid fluids, led to an increased hemodynamic stability. ${ }^{31,32}$ We chose to administer no fluids to be able to evaluate the hemodynamic response to SA with minimal effect on preload. Moreover, the administration of overzealous and unnecessary fluid can lead to complications such as bladder retention, pulmonary edema, and increased length of stay. ${ }^{33}$

Several limitations to our study should be noted. First, because our main focus was to study the hemodynamic effect of SA using only a finger cuff, the decision whether to allocate patients to the MD or LD group was left at the discretion of the attending anesthesiologist based on the patient characteristics and type of surgery due to patient safety. This may introduce a selection bias. However, after evaluation, baseline patient characteristics, types of surgery, and hemodynamic variables did not differ between the two dosage groups. However, small differences between patients such as frailty are not represented in "crude" scores such as American Society of Anaesthesiologists class and age. Although the range of sensory block was quite large, this is comparable with previous literature. ${ }^{9}$ A second limitation of this study is the lack of knowledge of the precision of Nexfin ${ }^{\mathrm{TM}}$. Although Nexfin ${ }^{\mathrm{TM}}$ has been shown to accurately provide $\mathrm{CO}$ measurement and show acceptable $\mathrm{CO}$ tracking capabilities, ${ }^{6,34}$ its precision is uncertain due to difficulties in previous studies evaluating this device, such as unknown precision of the reference technique, small sample sizes, specific patient study groups, and the studies' COs to determine accuracy range from very low to very high. ${ }^{35,36}$ This makes Nexfin ${ }^{\mathrm{TM}}$ possibly unsuitable to demonstrate subtle CO differences. However, Nexfin ${ }^{\mathrm{TM}}$ is the only $\mathrm{CO}$ measuring device that is adequately validated in a clinical setting and measures continuously and noninvasively, making it applicable to monitor awake patients.

\section{Conclusion}

Blood pressure decreased significantly after the onset of SA without fluid preloading in elderly patients. The hypotension that occurred was caused by a decrease in SV but not by a decrease in SVR. There was no difference in $\mathrm{CO}$ and blood pressure change between dosages of 10 or $15 \mathrm{mg}$ bupivacaine.

\section{Abbreviations}

$\mathrm{ABP}_{\mathrm{N}}$, noninvasive blood pressure measurement using Nexfin ${ }^{\mathrm{TM}}$; $\mathrm{CO}$, cardiac output; $\mathrm{CO}_{\mathrm{N}}$, noninvasive cardiac output measurement using Nexfin ${ }^{\mathrm{TM}}$; LD, low dose; MD, medium dose; MAP, mean arterial pressure; SA, spinal anesthesia; SV, stroke volume; SAP, systolic arterial pressure; SVR, systemic vascular resistance. 


\section{Disclosure}

The authors report no conflicts of interest in this work.

\section{References}

1. Carpenter RL, Caplan RA, Brown DL, Stephenson C, Wu R. Incidence and risk factors for side effects of spinal anesthesia. Anesthesiology. 1992;76(6):906-916.

2. Hartmann B, Junger A, Klasen J, et al. The incidence and risk factors for hypotension after spinal anesthesia induction: an analysis with automated data collection. Anesth Analg. 2002;94(6):1521-1529, table of contents.

3. Salinas FV, Sueda LA, Liu SS. Physiology of spinal anaesthesia and practical suggestions for successful spinal anaesthesia. Best Pract Res Clin Anaesthesiol. 2003;17(3):289-303.

4. Langesaeter E, Rosseland LA, Stubhaug A. Continuous invasive blood pressure and cardiac output monitoring during cesarean delivery: a randomized, double-blind comparison of low-dose versus high-dose spinal anesthesia with intravenous phenylephrine or placebo infusion. Anesthesiology. 2008;109(5):856-863.

5. Asehnoune K, Larousse E, Tadié JM, Minville V, Droupy S, Benhamou D. Small-dose bupivacaine-sufentanil prevents cardiac output modifications after spinal anesthesia. Anesth Analg. 2005;101(5):1512-1515.

6. Broch O, Renner J, Gruenewald M, et al. A comparison of the Nexfin ${ }^{\circledR}$ and transcardiopulmonary thermodilution to estimate cardiac output during coronary artery surgery. Anaesthesia. 2012;67(4):377-383.

7. Martina JR, Westerhof BE, van Goudoever J, et al. Noninvasive continuous arterial blood pressure monitoring with Nexfin ${ }^{\circledR}$. Anesthesiology. 2012;116(5):1092-1103.

8. Bogert LW, Wesseling KH, Schraa O, et al. Pulse contour cardiac output derived from non-invasive arterial pressure in cardiovascular disease Anaesthesia. 2010;65(11):1119-1125.

9. Hocking G, Wildsmith JA. Intrathecal drug spread. Br J Anaesth. 2004;93(4):568-578.

10. Carpenter RL, Caplan RA, Brown DL, Stephenson C, Wu R. Incidence and risk factors for side effects of spinal anesthesia. Anesthesiology. 1992;76(6):906-916.

11. Penáz J. Criteria for set point estimation in the volume clamp method of blood pressure measurement. Physiol Res. 1992;41(1):5-10.

12. Westerhof N, Lankhaar JW, Westerhof BE. The arterial Windkessel. Med Biol Eng Comput. 2009;47(2):131-141.

13. Aldrete JA, Kroulik D. A postanesthetic recovery score. Anesth Analg. 1970;49(6):924-934.

14. Critchley LA, Critchley JA. A meta-analysis of studies using bias and precision statistics to compare cardiac output measurement techniques. J Clin Monit Comput. 1999;15(2):85-91.

15. Ogawa T, Spina RJ, Martin WH, 3rd, et al. Effects of aging, sex, and physical training on cardiovascular responses to exercise. Circulation. 1992;86(2):494-503.

16. Lairez O, Ferré F, Portet N, et al. Cardiovascular effects of low-dose spinal anaesthesia as a function of age: an observational study using echocardiography. Anaesth Crit Care Pain Med. 2015;34(5):271-276.

17. Ferré F, Delmas C, Carrié D, Cognet T, Lairez O, Minville V. Effects of spinal anaesthesia on left ventricular function: an observational study using two-dimensional strain echocardiography. TurkJAnaesth Reanim. 2018;46(4):268-271.

18. Rooke GA, Freund PR, Jacobson AF. Hemodynamic response and change in organ blood volume during spinal anesthesia in elderly men with cardiac disease. Anesth Analg. 1997;85(1):99-105.
19. Guyton AC, Polizo D, Armstrong GG. Mean circulatory filling pressure measured immediately after cessation of heart pumping. Am J Physiol. 1954;179(2):261-267.

20. Lee JE, George RB, Habib AS. Spinal-induced hypotension: incidence, mechanisms, prophylaxis, and management: Summarizing 20 years of research. Best Pract Res Clin Anaesthesiol. 2017;31(1):57-68.

21. Priebe HJ. The aged cardiovascular risk patient. $\mathrm{Br} J$ Anaesth. 2000;85(5):763-778.

22. Fujiwara Y, Kurokawa S, Shibata Y, Asakura Y, Harado M, Komatsu T. Sympathovagal effects of spinal anaesthesia with intrathecal or intravenous fentanyl assessed by heart rate variability. Acta Anaesthesiol Scand. 2009;53(4):476-482.

23. Nakasuji M, Suh SH, Nomura M, et al. Hypotension from spinal anesthesia in patients aged greater than 80 years is due to a decrease in systemic vascular resistance. J Clin Anesth. 2012;24(3):201-206.

24. Monge García MI, Guijo González P, Gracia Romero M, et al. Effects of fluid administration on arterial load in septic shock patients. Intensive Care Med. 2015;41(7):1247-1255.

25. Valverde A, Gianotti G, Rioja-Garcia E, Hathway A. Effects of highvolume, rapid-fluid therapy on cardiovascular function and hematological values during isoflurane-induced hypotension in healthy dogs. Can J Vet Res. 2012;76(2):99-108.

26. Meyhoff CS, Hesselbjerg L, Koscielniak-Nielsen Z, Rasmussen LS. Biphasic cardiac output changes during onset of spinal anaesthesia in elderly patients. Eur J Anaesthesiol. 2007;24(9):770-775.

27. Donati A, Mercuri G, Iuorio S, et al. Haemodynamic modifications after unilateral subarachnoid anaesthesia evaluated with transthoracic echocardiography. Minerva Anestesiol. 2005;71(3):75-81.

28. Persichini R, Silva S, Teboul JL, et al. Effects of norepinephrine on mean systemic pressure and venous return in human septic shock. Crit Care Med. 2012;40(12):3146-3153.

29. Critchley LA, Stuart JC, Conway F, Short TG. Hypotension during subarachnoid anaesthesia: Haemodynamic effects of ephedrine. $\mathrm{Br} J$ Anaesth. 1995;74(4):373-378.

30. Moppett IK, Rowlands M, Mannings A, Moran CG, Wiles MD, Investigators N. LiDCO-based fluid management in patients undergoing hip fracture surgery under spinal anaesthesia: a randomized trial and systematic review. Br J Anaesth. 2015;114(3):444-459.

31. Oh AY, Hwang JW, Song IA, et al. Influence of the timing of administration of crystalloid on maternal hypotension during spinal anesthesia for cesarean delivery: preload versus coload. BMCAnesthesiol. 2014;14:36.

32. Ni HF, Liu HY, Zhang J, Peng K, Ji FH. Crystalloid Coload reduced the incidence of hypotension in spinal anesthesia for cesarean delivery, when compared to crystalloid preload: a meta-analysis. Biomed Res Int. 2017;2017:3462529.

33. Schol PB, Terink IM, Lancé MD, Scheepers HC. Liberal or restrictive fluid management during elective surgery: a systematic review and meta-analysis. J Clin Anesth. 2016;35:26-39.

34. Helmi M, de Wilde RB, Jansen JR, et al. Comparing hemodynamic effects with three different measurement devices, of two methods of external leg compression versus passive leg raising in patients after cardiac surgery. J Clin Monit Comput. 2013;27(2):163-170.

35. Geisen M, Ganter MT, Hartnack S, Dzemali O, Hofer CK, Zollinger A. Accuracy, precision, and trending of 4 pulse wave analysis techniques in the postoperative period. J Cardiothorac Vasc Anesth. 2018;32(2):715-722.

36. Saugel B, Cecconi M, Wagner JY, Reuter DA. Noninvasive continuous cardiac output monitoring in perioperative and intensive care medicine. Br J Anaesth. 2015;114(4):562-575. 


\section{Publish your work in this journal}

Local and Regional Anesthesia is an international, peer-reviewed, open access journal publishing on the development, pharmacology, delivery and targeting and clinical use of local and regional anesthetics and analgesics. The journal is included in PubMed, and welcomes submitted papers covering original research, basic science, clinical studies, reviews and evaluations, guidelines, expert opinion and commentary, case reports and extended reports. The manuscript management system is completely online and includes a very quick and fair peer-review system, which is all easy to use. Visit http://www.dovepress.com/ testimonials.php to read real quotes from published authors.

Submit your manuscript here: https://www.dovepress.com/local-and-regional-anesthesia-journal 